\title{
FORMULATION DEVELOPMENT AND CHARACTERIZATION OF TEA TREE OIL LOADED ETHOSOMES
}

\author{
Vijayan Venugopal*, Raymond Goh, Tan Yee Ping, Tan Jia Jin
}

Faculty of Pharmacy, AIMST

University, Kedah, Semeling, Malaysia-08100.

Submitted: $20-07-2015$

Revised: 05-09-2015

Accepted: 15-12-2015

*Corresponding author Vijayan Venugopal

Email:

vijayanv2@gmail.com

\begin{abstract}
To prepare ethosomes containing tea tree oil by hot homogenization method and to evaluate its physical characters and in vitro release pattern. The preformulation studies were carried out by standard procedure. The morphology of globule was studied by optical microscopy. The globule size and zeta potential was determined by Zetasizer, respectively and in vitro study was done by diffusion method and the drug content was analyzed by HPTLC method. The release kinetics was also studied by fitting into few mathematical models. All the formulations were showed spherical and unilamellar shape with globule size of 931 to $975 \mathrm{~nm}$, the zeta potential in the range of -40 to $-52 \mathrm{mV}$ and entrapment efficiency was 57 to $65 \%$. Finally the in vitro release studies showed the drug release from the ethosomal vesicles was burst release at initial time followed by sustained release over throughout the study. From the above consideration of evaluation studies, the tea tree oil loaded ethosomal formulation F5 shows best globule size, zeta potential and entrapment efficiency. The sustained action was confirmed by in vitro release studies. All the formulations are followed zero order drug release and diffusion type of mechanism of drug releases with Fickian model. Ethosome loaded tea tree oil could be the best choice for topical application.
\end{abstract}

Key words: tea tree oil, ethosomes, HPTLC, globule size, zeta potential

\section{INTRODUCTION}

Topical drug delivery system is considered to be one of the most relevant routes for treating skin diseases. Despite of having the advantages of self administration, patient compliance, and reduction in adverse effects systemically, this system has the limitation of slow diffusion across the stratum corneum (Touitou et al., 1996) and the barrier property of the skin limits the delivery of the drug through the skin (Patel et al., 2007). Therefore several approaches have been used to weaken this skin barrier and to enhance the administration of the drug through the skin. Among these strategies, novel preparation, such as liposomes was used for enhancing the transdermal permeation of many therapeutic agents. The vesicular system i.e. liposome in topical drug delivery system has been studied for many uses but the unstable nature limits their use at clinically and industrial levels. In order to improve the stability of liposomes concept, the proliposomes has been proposed. But because of poor skin permeability, leakage of drug, breaking of vesicles, aggregation and fusion of vesicles, these system is not much successful for effective transdermal drug delivery (Cevc et al., 1997).

Ethosomal vesicles have been found to be capable enough in enhancing permeation of topical agents to the deeper tissues through the stratum corneum (Touitou et al., 2001). Enhanced permeation through these vesicles is not only due to the presence of ethanol but also due to the fact that these vesicles are highly deformable and malleable that allows their better penetration across the skin (Jain et al., 2004). This system has good property to permeate intact through the human skin due to its high elasticity properties, which has an immense consequence for design of carrier system to be applied topically both for local and systemic delivery of hydrophilic and lipophilic drugs (Paolino et al., 2005).

Some of the studies showed that ethosomes have better penetration than other vesicular systems. Dayan and Touitou et al. compared the ethosomal system and classic 
liposomal system of trihexyphenidyl $\mathrm{HCl}$ and found that ethosomes dramatically enhanced the skin permeation (Dayan et al., 2000). Further transdermal delivery of cannabidiol by using ethosomal carrier was also designed by Lodzki and colleagues (Lodzki et al., 2003). Again Godin and Touitou (2004) investigated the dermal and intracellular delivery of bacitracin from ethosomes (Godin et al., 2004). After that Elsayed $e t$ al. studied the mechanism by which ethosomes can improve skin delivery of ketotifen (Elsayed et al., 2006).

Acne vulgaris is a disease of the pilosebaceous follicle characterized by noninflammatory (open and closed comedones) and inflammatory lesions (papules, pustules, and nodules). Its pathogenesis is multifactorial interplay of hormonal, bacterial, and immunological (inflammatory) factors results in the formation of acne lesions (Arora et al., 2011). Although acne is not a life-threatening condition, it can have detrimental effects on the quality of life of affected individuals. Although generally considered to be a benign, self limiting condition, acne may cause severe psychological problems or disfiguring scars that can persist for a lifetime. It is a pleomorphic disorder and can manifest at any time during life but it most commonly presents between ages of $12-24$, which estimates of $85 \%$ of population affected. In recent years multifactorial nature of acne has been elucidated (Bloom et al., 2004). Many natural agents are used for the acne treatment; some of them are produces itching and redness on the skin. Amir et al. investigated that tea tree oil can effectively used for the treatment of mild to moderate acne (Amir et al., 2007).

Extracted from the leaves of the tea tree, studies have confirmed tea tree oil's antibacterial activity against harmful microbes without damaging normal, healthy skin microbes (Johns et al., 1992). This includes inhibiting grow th of the gram positive bacteria associated with acne (Propionibacterium acnes). Using the topical route eliminates these side effects, increase patient compliance, target of the active ingredient for a local effect (Brophy et al., 1989). How ever, the insufficiency of skin permeation of tea tree oil formulations leads to low clinical efficiency. Thus, an improved ethosomal formulation with a high degree of permeation could be useful for the treatment of Acne.

\section{MATERIAL AND METHODS}

The tea tree oil was obtained from Integria Healthcare, Australia. Cholesterol was obtained from KLH chemicals, Malaysia. Lecithin and ethanol were purchased from Suka chemicals, SDN BHD, Malaysia. All other chemicals and solvents used were of analytical grade.

\section{Preparation of tea tree oil loaded ethosomes}

Ethosomal formulation was prepared according to the method reported by the Touitou et al., 2001. Various quantities of Soya Lecithin and cholesterol (Table I) were dissolved in required volume of ethanol and stirred well until completely dissolved (solution A). Required quantities of Tea tree oil was dissolved in ethanol by a vigorous stirring in a covered vessel at room temperature (solution B). Solution A and solution B was mixed thoroughly by using homogenizer at $6000 \mathrm{rpm}$ for $5 \mathrm{~min}$ and $50 \mathrm{~mL}$ of water was added in drop wise manner in the center of the vessel, which was homogenized for $15 \mathrm{~min}$ at $6000 \mathrm{rpm}$ in room temperature. Ethosomes were formed as cloudy homogeneous liquid. The formed tea tree oil loaded ethosomes were stored at $4^{\circ} \mathrm{C} \pm 0.5^{\circ} \mathrm{C}$ for further evaluation studies.

\section{Characterization of ethosomes Vesicle shape}

Ethosomes shape and size was measured by optical microscope connected with Dewinter microscopic software pro 4.1 version. A drop of ethosomal sample was placed in clean glass slide and focused by under 100x lens. The image was snapped by dewinter Digital camera.

\section{Vesicle size}

The vesicle size of formulated ethosomes was analyzed by using Malvern sizer version 7.1. Few drops of formulation were dispersed in distilled water and the sample was placed in clear disposable zeta cell at $25^{\circ} \mathrm{C}$ for $80 \mathrm{~s}$ and the reading was taken triplicate. 


\section{Zeta potential}

The charge of the ethosomes was determined by measuring the zeta potential by laser dropper anemometry using Zetasizer version 7.1. Ethosomes were diluted with distilled water and the samples were placed in the electrophoretic cell where the potential of $150 \mathrm{mV}$ was established and analysis was done at triplicate.

\section{Entra pment efficiency (EE)}

The entrapment efficiency of Tea tree oil loaded ethosomal vesicles was determined by ultracentrifugation method. The entrapment efficiency was determined after separating the unentrapped drug by centrifugation at 5000rpm for $2 \mathrm{~h}$. The vesicles were washed with water and analyzed for drug content by using HPTLC method at 366nm.n The EE was calculated triplicate.

\section{HPTLC analysis of tea tree oil}

In the proposed HPTLC method the reference standard (terpinen-4-ol) was spotted on to the pre-coated TLC plates (Brophy et al., 1989). The chromatographic development was performed using a mixture of toluene and ethyl acetate (85:15) as mobile phase with twodimensional development under the following conditions: chamber saturation time, $45 \mathrm{~min}$; temperature, $25^{\circ} \mathrm{C} \pm 2{ }^{\circ} \mathrm{C}$; migration distance, $80 \mathrm{~mm}$. After development, the TLC plates were dried completely. To visualize the zones, plates were sprayed with an anisaldehyde reagent and heated for $15 \mathrm{~min}$ at $105^{\circ} \mathrm{C}$. Quantification of terpinen-4-ol was achieved by scanning with CAMAG TLC scanner 3 (slit dimension, $4 \mathrm{~mm} \times 0.1 \mathrm{~mm}$; scanning speed $1 \mathrm{~mm} / \mathrm{s}$; wavelength of determination, $366 \mathrm{~nm}$ at absorbance mode) and the automated software produced the chromatogram by plotting absorbance against $\mathrm{Rf}$ values.

\section{Analysis of tea tree oil}

The tea tree oil was run on pre-coated TLC plates as described in HPTLC method above. Tea tree oil concentration was determined by quantifying the terpinen-4-ol content. The chromatogram was developed by scanning with CAMAG TLC scanner 3 (slit dimension, $4 \mathrm{~mm} \times 0.1 \mathrm{~mm}$; scanning speed
$1 \mathrm{~mm} / \mathrm{s}$; wavelength of determination, $366 \mathrm{~nm}$ at absorbance mode) and the automated software produced the chromatogram by plotting absorbance against Rf values.

\section{In vitro drug release studies}

The in vitro release of tea tree oil loaded ethosomes was carried out in the modified Franz diffusion cell at $37^{\circ} \mathrm{C} \pm 2{ }^{\circ} \mathrm{C}$. About $1 \mathrm{~mL}$ of ethosomes suspension was placed on dialysis membrane. The diffusion cell was filled with $7 \mathrm{~mL}$ of phosphate buffer ( $\mathrm{pH} 7.4)$. The donor compartment was clamped over it with the help of strings (Forster et al., 2009). About $1.5 \mathrm{~mL}$ of sample withdrawn at predetermined time intervals up to $12 \mathrm{~h}$. Fresh medium was replaced to the receptor compartment each time to maintain sink condition. The sample was analyzed by using HPTLC method at $366 \mathrm{~nm}$. The release data was subjected to kinetics profile to assess the mechanism of drug release.

\section{RESULTS AND DISCUSSION \\ Method preparation of Ethosome}

A total of six ethosomal formulations were prepared with varying concentration and volume of lecithin and ethanol, while the concentration and volume of tea tree oil containing the active component of interest (terpinen-4-ol) was kept constant. (Table I) Ethosomes were prepared as reported by Touitou et al., (2000). In brief soya lecithin in ethanol solution and tea tree oil in ethanol solution were homogenized by homogenizer. The fine droplets were emulsified by aqueous phase addition to form O/W type of emulsion. The emulsion globule size was stabilized by conteneous the homogenization. The beaker was covered with Para film or aluminum foil to avoid ethanol evaporation. The final stabilized ethosomes was kept at room temperature for $30 \mathrm{~min}$ under continuous stirring. Etha nol acts as a penetration enhancer through the skin. Ethanol penetrates into intercellular lipids and increases the fluidity of cell membrane lipids and decrease the density of lipid multilayer of cell membrane. The formulation compatibility was assessed by FTIR spectroscopy and the spectrum of tea tree oil loaded ethosomes was correlated with pure tea tree oil. 
Vijayan Venugo pal

Table I. Formulation of tea tree oil loaded ethosomes.

\begin{tabular}{ccccc}
\hline $\begin{array}{c}\text { Formulation } \\
\text { Code }\end{array}$ & $\begin{array}{c}\text { Lecithin } \\
(\mathbf{m g})\end{array}$ & $\begin{array}{c}\text { Ethanol } \\
(\mathbf{m L})\end{array}$ & $\begin{array}{c}\text { Tea tree oil(terpinen-4-ol) } \\
\text { (drops) }\end{array}$ & $\begin{array}{c}\text { Water } \\
\text { (mL) }\end{array}$ \\
\hline F1 & 100 & 40 & 8 & 50 \\
F2 & 200 & 40 & 8 & 50 \\
F3 & 300 & 40 & 8 & 50 \\
F4 & 400 & 40 & 8 & 50 \\
F5 & 200 & 60 & 8 & 50 \\
F6 & 400 & 60 & 8 & 50 \\
\hline
\end{tabular}

Each drop of tea tree oil contains 50 ng of terpinen-4-ol

Table II. Physiochemical evaluation of ethosomes

\begin{tabular}{lcccccc}
\hline Parameter & F1 & F2 & F3 & F4 & F5 & F6 \\
\hline Morphology & Spherical & Spherical & Spherical & Spherical & Spherical & Spherical \\
Globule size(nm) & $975.3 \pm$ & $955.5 \pm$ & $967.6 \pm$ & $974.6 \pm$ & $931.2 \pm$ & $964.6 \pm$ \\
& 2.5 & 0.78 & 1.49 & 0.09 & 1.66 & 1.26 \\
E E(\%) & $65.8 \pm$ & $70.5 \pm$ & $73.3 \pm$ & $75.3 \pm$ & $63.0 \pm$ & $57.2 \pm$ \\
& 0.3 & 1.06 & 0.21 & 0.67 & 1.19 & 1.82 \\
Zeta potential (mV) & -47.62 & -45.12 & -52.64 & 42.17 & -40.49 & -46.45
\end{tabular}

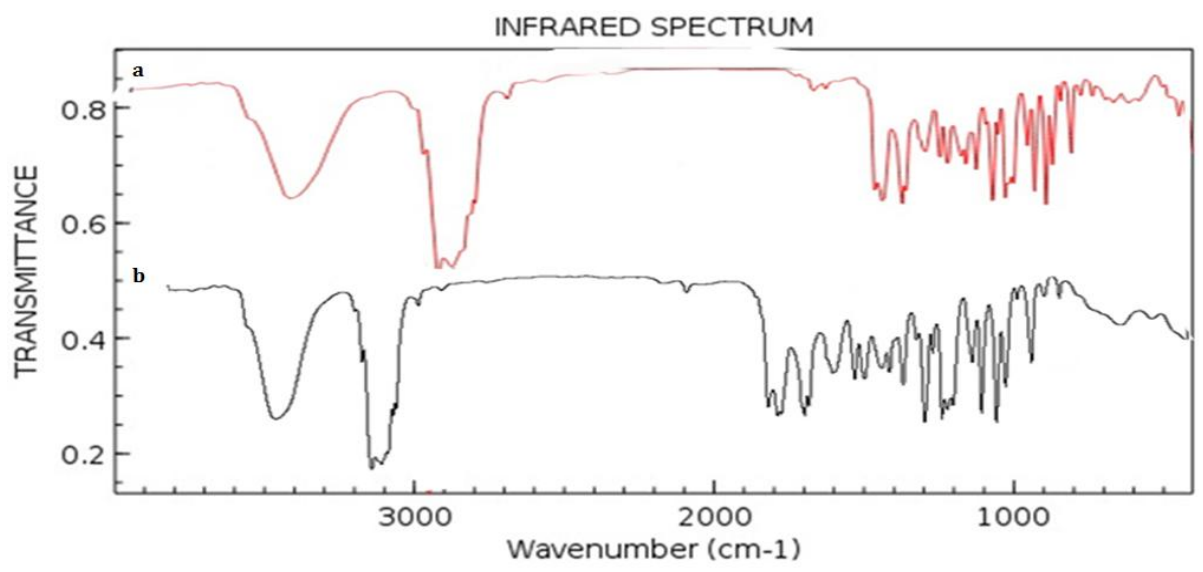

Figure 1. FTIR spectrum of pure tea tree oil (a) and oil loaded ethosomes(b)

Significant peaks of tea tree oil were observed at: $\mathrm{O}-\mathrm{H}(\mathrm{s})$ at $3362 \mathrm{~cm}^{-1}$, methyl group $\left(-\mathrm{CH}_{3}\right)$ observed at 2855 and $2924 \mathrm{~cm}^{-1}, \mathrm{C}-\mathrm{O}(\mathrm{s})$ bond 1025 and $1045 \mathrm{~cm}^{-1}$ and isopropyl group at $1368 \mathrm{~cm}^{-1}$. The spectral studies show that tea tree oil was compatibility with other ingredient (Figure 1). The ethosomes vesicles preparation was tested the morphology, globular size, entrapment efficiency, zeta potential and in vitro drug release.

\section{Morphology}

The morphology of ethosomal formulations were analyzed by optical microscopy connected with digital camera. The morphology of the ethosomes in all six formulations was found to be the uniform spherical and unilamellar shape (Figure 2). This shows that the concentration and volume of lecithin as well as ethanol does not affect much in the case of morphology of the ethosomal 


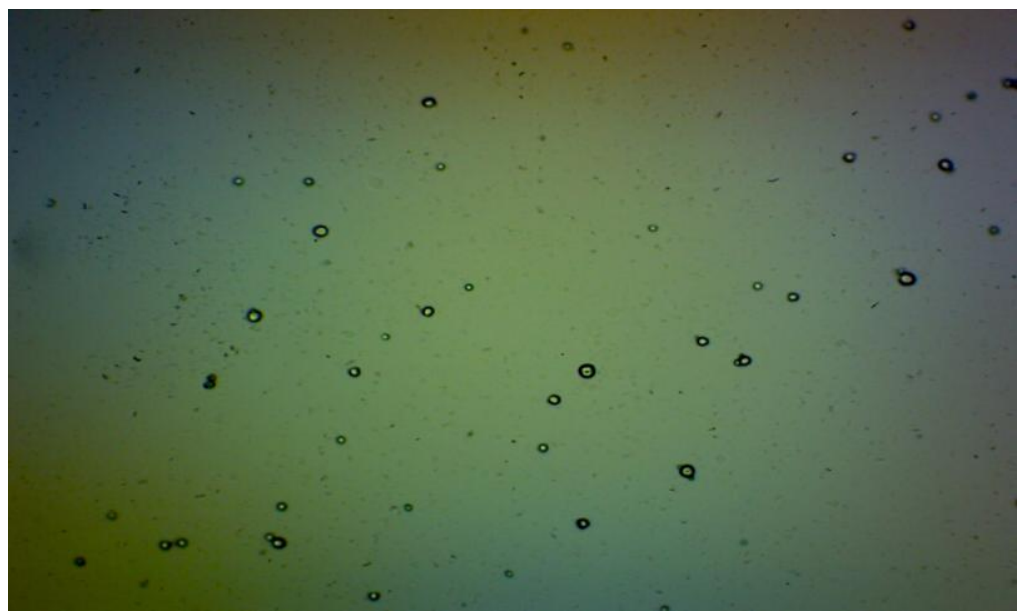

Figure 2. Optical microscopy image of ethosome

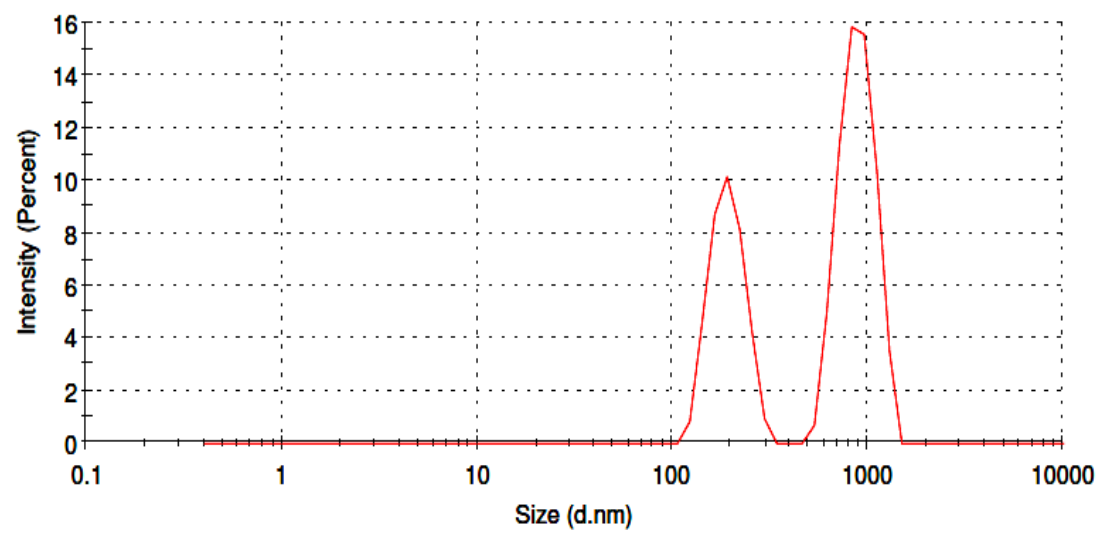

Figure 3. Globule size distribution graph

vesicles. However there are studies that found ethanol to cause the thinning of the outer membrane of the vesicle.

\section{Globule size}

The globular size of the vesicles varies from formulation to formulation, with the largest globular size being $975.3 \pm 2.5 \mathrm{~nm}$ from formulation 1, while the smallest globular size is $931.2 \pm 1.66 \mathrm{~nm}$ from formulation 5 (Figure 3). The size obtained does not seem to follow any kind of pattern in accordance with the increase and decrease of volume and concentration of lecithin and/or ethanol, though studies show that the size of vesicles increases with the increase in concentration of lecithin (Godin et al., 2004). Ethosomes are spherical vesicles with lamella extending throughout the entire volume of vesicles to the core (Godin and
Touitou, 2004). This lamellarity of ethosomal vesicles along with the ethanol allows the better solubility of both lipophilic and hydrophilic molecules. This might be the reason for the increased entrapment efficiency of ethosomes when ethanol concentration was increased. Ethanol at increased concentration was found to have decreasing effect on size. This may be due to solubilisation of lipids in ethanol or by the formation of hydrocarbon phase with interpenetrating properties due to of interpenetration, the size decreases.

\section{Zeta potential}

The zeta potential of formulated ethosomes was evaluated by zetasizer. Zeta potential is a key factor to evaluate the stability of colloidal dispersion. In general, globules 


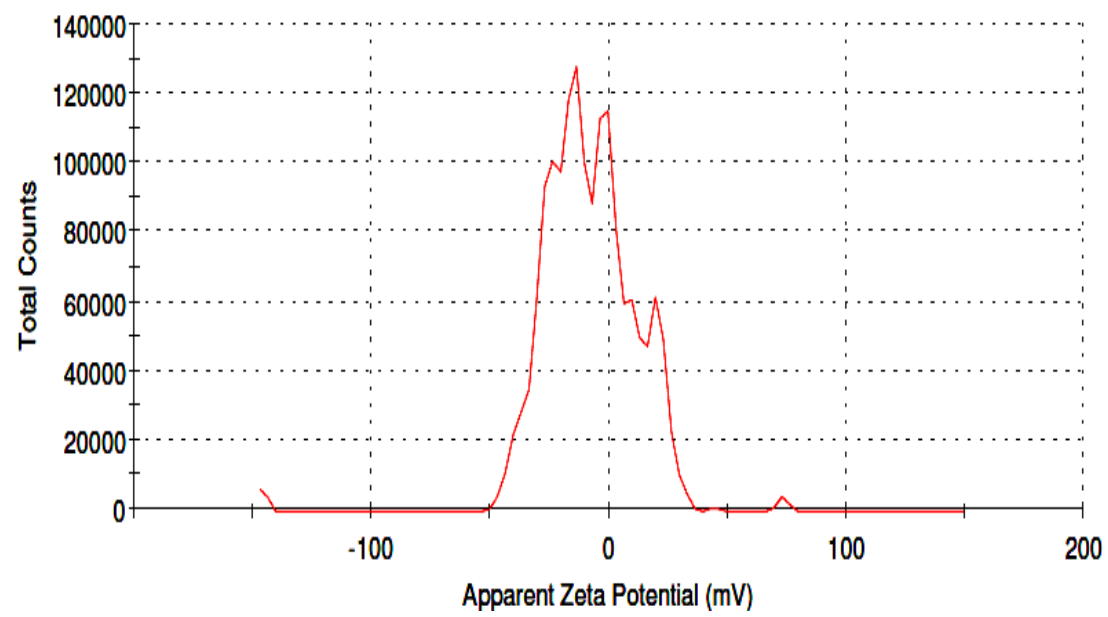

Figure 4. Zeta potential graph of ethosomes

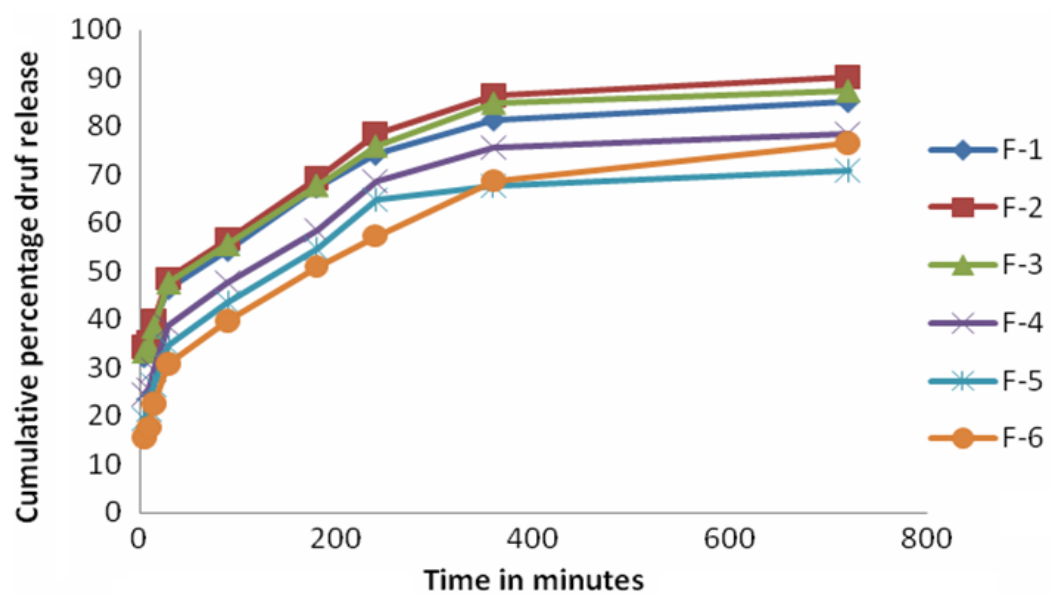

Figure 5. In vitro drug release profile of tea tree oil loaded ethosomes

could be dispersed stabilized when value of zeta potential was in the range of -40 to $+40 \mathrm{mV}$ which are due to electrical repulsion between particles formulation 5 was found to have the lowest value of zeta potential at $-40.49 \mathrm{mV}$ (Figure 4) while formulation 3 have the highest value at $-52.64 \mathrm{mV}$. The formulation F5 shows the best zeta potential compared to other formulations.

\section{Entrapment efficiency}

Entrapment is expected to increase with the increase in the amount of lecithin. Generally hydrophilic drugs are entrapped in the aqueous core inside lipid layer while the lipophilic drugs are retained in lipid lamella (Gupta et al., 2012). Encapsulation of a hydrophilic drug depends on the captured aqueous volume and that of lipophilic drug depends on the number of bilayers of lipid. The formulation with the highest entrapment efficiency was in the formulation 4 at $75.3 \pm 0.67 \%$ while the formulation with the least entrapment was in formulation 6 at $57.2 \pm 1.82 \%$. In this case a relationship between the increase in lecithin concentration and volume with increase in the entrapment of the active component terpinen-4-ol was observed. However when the concentration and volume of ethanol was increased, it retards the entrapment as compared to lower ethanol volume and concentration in formulation.

\section{In vitro drug release}

There is a clear evidence of increased drug diffusion into the skin with increased 
concentration of ethanol. There is increased diffusion with decreased vesicle size. This is because of the increased surface area available for diffusion. In order to find out the in vitro drug release of the ethosomes, readings were taken at specific time intervals for up to 12h. The withdrawn samples were analyzed by HPTLC method at $366 \mathrm{~nm}$ (Biju et al., 2005). The quantity of drug release in all the formulations (F1-F6) of ethosomal formulations were showed bust release upto $34 \%$ at initially $5 \mathrm{~min}$. From this, it is obvious that the decreased percentage of drug release was due to the more compact wall around the drug by the biodegradable lipid lecithin matrix and it signifies that they possess a sustained drug release for a prolonged period of time in all the formulations. The highest amount of drug released after $12 \mathrm{~h}$ is $90.35 \%$ from formulation 2, while the lowest is $70.89 \%$ from formulation 5 (Figure 5). Being soft and flexible, the ethosomes can penetrate easily in to the deeper layers of skin. Ethanol being an aliphatic chain disrupts the stratum corneum of skin by extracting the lipid (Jain et al., 2011). There is enhanced transdermal drug diffusion due to the presence of lipid bilayer in the vesicle structure, which is similar to the structure of skin. Lipids can also penetrate into the skin by follicular transport pathway and hence there is an enhanced diffusion with increased concentration of the lecithin in ethosomes. From that F5 shows sustained drug release over other formulations.

\section{In vitro release kinetic studies}

The in vitro release data was fitted into various kinetic equations i.e., zero order, first order, Higuchi and Korsemayer. The release constant was calculated from the slope of appropriate plot and the regression $\left(\mathrm{r}^{2}\right)$ was calculated. In the Insulin polymeric nanoparticle preparations of all formulations (F1-F6), the in vitro release kinetic was best fitted by zero order equation and the plots showed the highest linearity $\left(\mathrm{r}^{2}=0.7172\right.$ to $0.766)$ followed by first order $\left(r^{2}=0.7866\right.$ to $0.92)$ and Higuchi equation $\left(\mathrm{r}^{2}=0.8459\right.$ to 0.8905). Hence the drug release kinetics demonstrated that the concentration was nearly independent of drug release. The release exponent $\mathrm{n}=0.59$, which appears to be showing anomalous diffusion. Release kinetics are often used for comparative purposes and relating the release parameters with bioavailability. It is also used to study the influences of formulation factors on the drug release for optimization as well as control of drug release from ethosomes. The in vitro release data was subjected to zero order, first order, Higuchi and KorsmeyerPeppas model to establish the drug release mechanism and kinetics of drug release from ethosomes. When the data was subjected to zero order and first order kinetic model, a linear relationship was observed with high $\mathrm{r}^{2}$ values for zero order as compared to first order model; this suggests that the ethosomes were zero order controlled release of drug from their ethanolic carrier. Higuchi's model $\mathrm{r}^{2}$ values suggest that the drug release from ethosomes follows diffusion mechanism. The exact release mechanism was analyzed by Korsmeyer-Peppas model. The values of ' $n$ ' obtained for all the ethosomal formulations was $\geq 0.2$ to $\leq 0.5$, thus showing that the drug release followed the Fickian anomalous diffusion mechanism ${ }^{32}$.

\section{CONCLUSION}

The research work is deals with the study carried out by the writer for the past one year on the formulation and evaluation of tea tree oil loaded ethosomal formulations. Researcher has developed many formulations in order to reduce the burden of the complications. We tried with new approach of ethosomal formulation to might be increase the penetration of tea tree oil into deeper skin. The tea tree oil loaded ethosomes was prepared by using hot homogenization and subjected to globule size, morphology, zeta potential, entrapment efficiency and in vitro drug release studies. All the formulations were showed spherical and unilamellar shape with globule size of 931 to $975 \mathrm{~nm}$, the zeta potential in the range of -40 to $-52 \mathrm{mV}$ and entrapment efficiency was 57 to $65 \%$. Finally the in vitro release studies showed the drug release from the ethosomal vesicles was bust release at initial time followed by sustained release over throughout the study. From the above consideration of evaluation studies, the tea tree oil loaded ethosomal formulation F5 shows best globule size, zeta potential and entrapment efficiency. The sustained action was confirmed 
by in vitro release studies. All the formulation followed zero order drug release and diffusion type of mechanism with Fickian model. In our present investigation, the prepared ethosomes with smaller vesicle size and maximum oil entrap, which makes it suitable delivery system for topical application. In acne conditions there is a need to deliver the drug to deeper layers. This is possible with ethosomes which release drug at various points along the penetration pathway.

\section{ACKNOWLEDGMENTS}

The authors are acknowledged AIMST University, Malaysia for financial support of this research.

\section{REFE RE NCES}

Arora MK., Yadav A., Saini V., 2011. Role of hormones in acne vulgaris. Clin. Biochem. 44: 1035-1040.

Bloom DF., 2004. Is acne really a disease? A theory of acne as an evolutionarily significant, high-order psychoneuroimmune interaction timed to cortical development with a crucial role inmate choice. Medical Hypotheses. 62: 462-469.

Biju SS., Alka A., Rafiullah MRM., Roop K., Khar A., 2005. Validated HPTLC method for determination of tea tree oil from cosmeceutical formulations, $J$. Pharm. Biom.An.. 38: 41-44.

Brophy JJ., Davies NW., Southwell IA., Stiff IA., Williams LR., 1989. Gas chromatographic quality control for oil of melaleuca terpinen-4-ol type (Australian tea tree). J Agric Food Chem. 37: 1330-1335

Cevc G., Blume G., Schatzlein A., 1997. In vivo Transfersomes mediated transepidermal delivery improves regiospecificity and biological activity of corticosteroids. J Control Release. 45: 211226.

Dayan N., Touitou E., 2000. Carriers for skin delivery of trihexyphenidyl $\mathrm{HCl}$ : Ethosomes vs. liposomes. Biomaterials. 21: 1879-1885.

Elsayed MM., Abdallah OY., Naggar VF., Khalafallah NM., 2006. Deformable liposomes and ethosomes: mechanism of enhanced skin delivery. Int J Pharm. 322: 60-66.

Forster M., Bolzinger MA., Fessi H., Briancon S., 2009. Topical delivery of cosmetics and drugs. Molecular aspects of percutaneous absorption and delivery. Eur. J. Dermatology. 19: 309-323

Godin B., Touitou E., 2004. Mechanism of bacitracin permeation enhancement through the skin and cellular membranes from an ethosomal carrier. J Control Release. 94: 365-379.

Gupta NB., Loona S., Khan MU., 2012. Ethosomes as elastic vesicles in transdermal drugdelivery: an overview. Int. J.Pharm. Sci. Res. 3: 682-687.

Heeremans JLM., Gerristen HR., Meusen SP., Mijnheer FW., Gangaram RS., Panday G., Prevost R., Kluft C., Crommelin DJA., 1995. The preparation of tissue type plasminogen activator ( $\mathrm{t}-\mathrm{PA}$ ) containing liposomes: entrapment efficacy and ultracentrifugation damage. J Drug Target. 3: 301.

Ho V., Schacter D., Miller R., 1995. Acne management for the 90s: current treatment guidelines. The Canadian Journal of Diagnosis. 12: 1-25.

Jain H., Patel J., Joshi K., Upadhyay UM., 2011. Ethosomes: a novel drug carrier. Pharmacie Globale. Int. J.Comprehensive Pharmacy. 2: 1-14.

Jain S., Umamaheshwari RB., Bhadra D., Jain NK., 2004. Ethosomes: a novel vesicular carrier for enhanced transdermal delivery of an anti - HIV agent, Ind J Pharma Sci. 66: 72-81.

Johns MR., Johns JE., Rudolph V., 1992. Steam distillation of tea tree (Melalenca alternifolia) oil. J Sci Food Agric. 58: 49-53

Lodzki M., Godin B., Rakou L., Mechoulam R., Gallily R., Touitou E., 2003. Cannabidiol-transdermal delivery and anti-inflammatory effect in a murine model. J Control Release. 93: 377-387.

New RRC. 1990. Size determination of liposomes, Liposomes - A practical approach. Oxford University Press, New York. pp.154.

Paolino D., Lucania G., Mardente D., Alhaique F., Fresta M., 2005. Ethosomes for skin 
delivery of ammonium glycyrrhizinate: In vitro percutaneous permeation through human skin and in vivo anti-inflammatory activity on human volunteers. J Control Release. 106: 99-110.

Patel S., 2007. Ethosomes: A promising tool for transdermal delivery of drug. Pharma Info. Net. 5: 1-7.

Touitou E., 1996. Composition of applying active substance to or through the skin. US patent 5540 934. July 30.
Touitou E., Dayan N., Bergelson L., Godin B., Eliaz M., 2000. Ethosomes novel vesicular carriers for enhanced delivery: characterization and skin penetration properties. J Control Release. 65: 403-418.

Touitou E., Godin B., Dayan N., Weiss C., Piliponsky A., Levi - Schaffer F., 2001. Intracellular delivery mediated by an ethosomal carrier. Biomaterials. 22: 30533059. 